\title{
Making the snip hip: mass circumcision in Rwanda
}

$\mathrm{T}$ he goal was ambitious: halve HIV transmissions within a country in which male circumcision was virtually unheard of by convincing some two million men to volunteer for the procedure. But a successful six-month mass circumcision pilot in two districts has Rwandan officials and health experts predicting that the tiny African nation will easily achieve that goal.

Minister of Health Dr. Agnes Binagwaho estimates Rwanda will reach the two million target by 2013, perhaps sooner if the World Health Organization (WHO) approves the use of a new nonsurgical device called the PrePex system that purportedly enables bloodless male circumcision to be performed in nonsterile settings while eliminating the need for anaesthesia and sutures.

A federally sponsored 2010 study, which found the device to be both safe and effective, was part of some three-year's preparations by the Rwandan government to promote male circumcision and build the health infrastructure necessary for the rollout of a national voluntary circumcision drive this summer.

So far, it's these preparations that have set Rwanda apart from other African nations that have been less successful in pushing male circumcision as an HIV prevention tool, says Dr. Jennifer Mbabazi, in charge of biomedical prevention at the Rwanda Center for Treatment and Research on AIDS.

"People once feared that if you were circumcised, your sexual drive would go down, your penis would atrophy or your wife would become promiscuous," says Mbabazi. "In Rwanda, that attitude has changed dramatically. We now have people who are willing and ready to be circumcised. What remains is our ability to serve them."

Mass circumcision campaigns have been launched in various sub-Saharan countries since the WHO and the Joint

Rwanda held back in favour of building the necessary public interest and medical capacity to take its mass circumcision campaign countrywide.

Top leaders, including President Paul Kagame, have preached the benefits of male circumcision. The Rwandan military has implemented its own highly publicized voluntary circumcision program. And specialized training for medical professionals has decreased the rate of botched circumcisions, which Mbabazi explains have been the Achilles heel of hastier efforts.

Rwanda's slow and steady approach appears to be paying off, she says. More than 5000 men were circumcised during the recently concluded six-month pilots in Nyanza and Ruhengeri districts. Demand for the procedure outstripped supplies of disposable circumcision kits at some sites, where crowds of hundreds waited patiently, in some cases up to a week, to undergo what was once considered a taboo practice.

"We're also starting to see male circumcision as an entry point for men coming into the hospitals for other family planning and reproductive

United Nations Programme on HIV/AIDS in 2007 began promoting male circumcision as a means of combating HIV. The recommendation relies on studies suggesting that circumcised men reduce their risk of infection by about $60 \%$, explains Binagwaho.

But stigma and myths surrounding the foreign procedure, as well as a lack of medical infrastructure and trained professionals to perform it, have hobbled hasty efforts to implement smaller circumcision campaigns elsewhere, says Mbabazi.

Typical of its nationwide-or-bust approach to health intervention, health services, in particular for HIV testing," Mbabazi says.

Adult circumcision is particularly in vogue among Rwandan males in their teens and twenties, Mbabazi says, although the reason for the trend is not yet clear. Partly, it may be attributable to the fact that many Rwandan young adults were exposed to another culture, having grown up outside of the country after fleeing with their families during the 1994 genocide.

The "tough guy" picture painted by the military's endorsement of male circumcision doesn't hurt either, she quips.

The switch in public sentiment has 
been too extreme in some cases, she adds, as the government and health professionals have had to dispel new myths that male circumcision is a kind of "magic bullet" or "invisible condom" against HIV.

Other wrinkles in Rwanda's mass circumcision campaign will hopefully be ironed out by WHO approval of the PrePex system, says Binagwaho.

The device works through an elastic mechanism that fits closely around an inner ring, trapping the foreskin, which dries up and is removed after a week. The result: a relatively "painless" male circumcision that can be performed in nonsterile settings by nonmedical health workers with no significant adverse effects (www.retroconference .org/2011/Abstracts/40740.htm).

The PrePex system is already approved by the European Union, says Binagwaho, and would save time, money and human resources in African nations where health infrastructure and funding are limited.

"We cannot go with the surgical circumcision as far as we would like to," she says. "With WHO approval of the device, we could perform male circumcisions in classrooms over the weekend or run mobile clinics out to remote communities, rather than take up precious hospital beds or force men to travel for the circumcisions."

Enabling nonmedical health workers to perform circumcisions would also relieve the burden of the national program on doctors, who are currently expected to incorporate male circumcision into routine health services they provide and do not receive extra compensation for the time it takes to perform the procedures or follow up with patients, Mbabazi says.

A new study sponsored by the Rwandan government is currently underway to compare the PrePex system to surgical circumcision for use in resource-limited settings, with a particular focus on cost-effectiveness. Lauren Vogel, CMAJ

CMAJ 2011. DOI:10.1503/cmaj.109-3950 